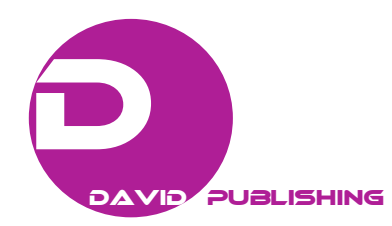

\title{
Optimization of Manufacturing Supply Chain with Stochastic Demand and Planned Delivery Time
}

\author{
Sadok Turki and Nidhal Rezg \\ LGIPM, Université de Lorraine, Ile du Saulcy, 57045 Metz Cedex, France
}

\begin{abstract}
In this paper, a manufacturing supply chain system composed by a single-product machine, a buffer and a stochastic demand is considered. A stochastic fluid model is adopted to describe the system and to take into account stochastic delivery times. The objective of this paper is to evaluate the optimal buffer level used in hedging point policy taken into account planned delivery times, machine failures and random demands. This optimal buffer allows minimizing the sum of inventory, transportation, lost sales and late delivery costs. Infinitesimal perturbation analysis method is used for optimizing the proposed system. Using the stochastic fluid model, the trajectories of buffer level are studied and the infinitesimal perturbation analysis estimators are evaluated. These estimators are shown to be unbiased and then they are implanted in an optimization algorithm, which determines the optimal buffer level in the presence of planned delivery time. Also in this work, we discuss the advantage of the use of the infinitesimal perturbation analysis method comparing to classical simulation methods.
\end{abstract}

Key words: Manufacturing supply chain, stochastic fluid model, infinitesimal perturbation analysis, planned delivery time.

\section{Introduction}

Stochastic fluid model [1-4] is widely used to control, analyze, and improve the performance of manufacturing supply chain systems. This model has been long adopted as a modeling technique of queuing theory, for analysis and synthesis of discrete event systems [5, 6]. Many researchers use stochastic fluid model for optimizing and evaluating supply chain systems, due to the fact that this model is simple to study; it makes the performance analysis efficient without the need to track part by part and hence allowing focusing on important events such as machine failures and inventory full/empty. Furthermore, stochastic fluid model is considered as a very useful model for simulating manufacturing supply chain systems [7, 8]. Tzenova et al. [9] used a stochastic fluid model for analyzing and modelling a system composed by a single server and $K$ separate infinite capacity buffers, the objective is to study the output process of multiclass fluid models with static

Corresponding author: Sadok Turki, associate professor, Ph.D., research fields: logistics, and optimization of production systems. E-mail: sadok.turki@univ-lorraine.fr. priorities. In this paper, we will consider a continuous flow manufacturing supply chain system, and then this system can be easily modeled by using stochastic fluid model. Furthermore, using such a model, the IPA (infinitesimal perturbation analysis) [10-12] method can be invoked to optimize the proposed system. IPA is a well-developed approach that has been used to determine unbiased gradient estimators. Therefore, the use of stochastic fluid model allows opening up the possibility to derive unbiased estimators. In this paper, stochastic fluid model is adopted to describe the system and to take into account random delivery times.

Nowadays, many industrial companies are working to optimize their manufacturing supply chain system in order to produce and to deliver in time. These companies are working to reduce transportation delays such as the delivery time [13-16], which is the period of time that the product takes between a manufacturing warehouse and a costumer. Furthermore, these companies are proposing planned delivery time as a marketing strategy to attract customers. In this paper, we consider the delivery 
times as a random variable. Indeed, the producer proposes for customers a fixed delivery time called planned delivery time. By a planned delivery time, we mean the time from a customer's order until the due date. However, if the products do not arrive on or before the planned delivery time (late delivery of products), these products are accepted by the customer, but are penalized with a late delivery penalty. In this paper, we will investigate how to integrate and to model the random delivery time in the stochastic fluid model while preserving its simplicity and analyticity. Thereafter, we will determine the optimal buffer level taking into account random delivery times and machine failures, in order to minimize the inventory, transportation, lost sales and late delivery costs. Under this stochastic fluid model, a stochastic optimization problem will be formulated what aims to minimize the total expected cost via a simulation based optimization.

IPA $[8,17]$ is proposed in this study. Indeed, IPA is a technique, which allows estimating the gradients of a random variable with respect to some parameters of interest (e.g., buffer level, production rate, etc.). Then, these gradients can be used in stochastic approximation algorithms for determining the optimal parameter setting. Ho et al. [18] used a PA (perturbation analysis) method for determining the optimal allocation of buffers in a serial production line. The PA method was applied at the beginning of $1980 \mathrm{~s}$ in the domain of discrete event systems; indeed, the event-driven dynamics give rise to state trajectories (sample paths) from which one can very efficiently and no intrusively extract sensitivities of various performance metrics with respect to at least certain types of design or control parameters. Furthermore, the PA was used as a technique for the performance evaluation of discrete event systems through information obtained in a single simulation run [19-21]. This has led to the development of a theory of PA in discrete event systems. Besides, the most successful kind of PA is when the perturbation of parameters is infinitesimal. This kind of PA theory is IPA which is mostly used due to its ease of implementation and simplicity.

Nowadays, IPA is essentially applied to stochastic fluid models [22]. Markou and Panayiotou [23] applied the IPA method to stochastic fluid model and determined the estimators of the performance metrics of interest with respect to the buffer size. The authors used these estimators in a stochastic approximation algorithm to control the buffer size of the nodes of a cellular network to optimize the overall network's performance. Panayiotou and Cassandras [24] determined the optimal capacities (or hedging points) of the finished goods and work-in-process buffers for minimizing a cost function. They estimated the gradient of the cost function. Also, Markou and Panayiotou [23] investigated the implementation of various IPA estimators that have been derived based on a stochastic fluid model for the optimization of parameters (buffer size) of a discrete event system. Yao and Cassandras [25] used the IPA method for deriving the gradient of the performance metrics of interests with respect to the lot-size parameters. The authors proved that these estimators are unbiased before using them in an algorithm to obtain optimal lot sizes. Indeed, the unbiasedness is the principal condition for making the application of IPA useful in practice, since it enables the use of the sample IPA derivative in control and optimization methods that employ stochastic gradient-based techniques. Then, these estimators could be used in stochastic approximation algorithm. Another kind of the PA method called FPA (finite perturbation analysis) method [26, 27].

The contribution of this paper is to apply IPA method on stochastic fluid model with planed delivery time in order to derive unbiased gradient estimators of the cost function. To the best of our knowledge, there is no study that applies the IPA method to such system. Therefore, the consideration of system with planed delivery time combined with IPA method is a very 
recent topic, and the few existing results indicate that the problem may become challenging, as the IPA derivatives are more complicated than those that would be obtained for the system without planed delivery time.

The paper is organized as follows. The stochastic fluid model with planned delivery time is presented in Section 2. In Section 3, the IPA method is applied on the stochastic fluid model. Numerical results are presented in Section 4. Finally, the last section concludes the paper and gives some perspectives to our work.

\section{Manufacturing Supply Chain System with Planned Delivery Time}

The studied manufacturing supply chain system is composed by a single-product machine $M$, a buffer $B$ and a customer who demands at every time $t$ a quantity of products denoted by $d(t)$. We note that the demand $d(t)$ is random and is generated by a uniform distribution (for the demand event) and truncated normal distribution (for the demand value). The quantity of products outgoing from the buffer $B$ at time $t$, are transported to the customer and take a delivery time, denoted as $\tau(t)$, to arrive to the customer. The producer proposes for the customer a constant planned delivery time denoted as $\tau_{p l}$. The transported products can arrive to the customer before the end or exactly on time (i.e., $\tau(t) \leq \tau_{p l}$ ), or can arrive to the customer after the end of $\tau_{p l}$ (i.e., $\left.\tau(t)>\tau_{p l}\right)$. The number of products transported between the buffer and the customer at time $t$ is denoted by $g(t)$ (Fig. 1).

We assume that the machine is never starved. The machine $M$ is either up or down. The state of the machine at time $t$, denoted $\alpha(t)$, is given by:

$$
\alpha(t)= \begin{cases}1 & \text { if machine is up } \\ 0 & \text { if machine is down }\end{cases}
$$

When the machine is up, the production rate of $M$, denoted by $u(t)$, could take a value between 0 and its maximum rate $U$, i.e., $0 \leq u(t) \leq U$. When the machine is down $u(t)=0$. The times to failure and times to repair are random. The failure/repair process is an independent random process. It does not depend on the system parameters. We denote by $x(t)$ the buffer level at time $t$. Furthermore, we assume in this paper that:

- If the demand is unsatisfied, the demand is lost with a corresponding cost (lost sales cost);

- For building the buffer $B$ and to avoid having always unsatisfied demands, we assume that the maximal production rate permits to satisfy the demand, i.e., $U \geq d(t) \forall t$;

- At time $t=0$, we suppose that we have enough parts in the buffer to satisfy the first demand, i.e., $x(0) \geq$ $d(0)$;

- The delivery time $\tau(t)$ is random and strictly greater than zero $(\tau(t)>0)$;

- We assume that, the average system rate is larger than the demand.

Remark 1: In the first step of the approach for determining the optimal buffer level, we suppose that the capacity of buffer $B$ is infinite, and then the optimal buffer level will be determined according to the hedging point.

The possible events at every time $t$ are: machine failure (PM), repair (RM), buffer full (when the buffer level equals to the optimal buffer level) (SS) which occurs at time instant $t_{s s}$, buffer empty, i.e., $x(t)=0(\mathrm{SV})$ and demand (DE).

$u(t) \quad x(t)$

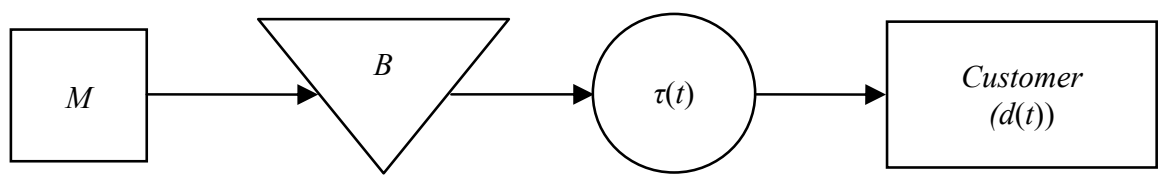

$g(t)$

Fig. 1 Studied manufacturing supply chain system. 
Assumption 1: For application of IPA and to avoid important discontinuities, we have to consider only one event at a time, thus we propose priorities between events if different events occur at the same time. The priority is assigned in a decreasing order as follows:

- buffer event (SS or SV);

- machine event (RM or PM);

- demand event (DE).

Assumption 2: We assume that the cost function is differentiable in $[0, \infty)$.

We denote by $x(t)$ the buffer level at time $t$. The number of parts in the buffer depends on the production speed $u(t)$ and the demand $d(t)$ :

$$
\frac{d x(x)}{d t}=u(t)-d(t)
$$

In this paper, we choose hedging point policy [28, 29] as a control policy, which ensures that the part does not exceed a given number of products, denoted by $h$. Furthermore, the hedging point has been proved to be the optimal policy for a one-product manufacturing system [30]. The Kanban policy [31] is generally applied to repetitive and quasi-continuous productions, this policy cannot respond quickly when the demand changes.

This control policy is defined as follows:

$$
u(t)= \begin{cases}d(t) & \text { if } \alpha(t)=1 \text { and } x(t)=h \\ U & \text { if } \alpha(t)=1 \text { and } x(t)<h \\ 0 & \text { if } \alpha(t)=0 \text { or } x(t)>h\end{cases}
$$

The number of unsatisfied demands (lost) per unit time is denoted by $D^{0}(t)$, and depends on the customer demand and the buffer level. Indeed, when the customer orders a demand $d(t)$ and the buffer is empty, the demand will not be satisfied at all and will be lost. Therefore, $D^{0}(t)$ is null if the buffer level is positive and is equal to the demand if the buffer is empty. The number of unsatisfied demands per unit time is defined as follows:

$$
D^{0}(t)= \begin{cases}0 & \text { if } x(t)>0 \\ d(t) & \text { if } x(t)=0\end{cases}
$$

The number of unsatisfied demands at time $t$ denoted by $L(t)$ is given by:

$$
\begin{cases}\frac{d L(t)}{d t}=D^{0}(t)=d(t) & \text { if } x(t)=0 \\ L(t)=0 & \text { if } x(t)>0\end{cases}
$$

Remark 2: We defined $L(t)$, because we will need it for writing the cost function. Indeed, $L(t)$ is number of unsatisfied demands at time $t$ and $D^{0}(t)$ is the number of unsatisfied demands (lost) per unit time.

The number of transported products is equal to the number of products outgoing from the buffer at time $t-\tau(t)$ and which will arrive to the customer at time $t$. Indeed, the speed of the number of products transported is equal to the demand per unit time $(d(t))$ minus the number of the unsatisfied demands per unit time $\left(D^{0}(t)\right)$.

The number of products transported at time $t$ is defined as follows:

$$
g(t)=\int_{t-\tau(t)}^{t}\left(d(t)-D^{0}(T)\right) d t
$$

The number of products arriving after the planed delivery time at time $t$ is indicated as $r(t)$, and is equal to the number of products transported at time $t$ when $\tau(t)>\tau_{p l}$

The number at time $t$ of products arriving after the planned delivery time (late delivery of products) is given by:

$$
r(t)=1 \cdot\left[\tau(t)>\tau_{p l}\right] \cdot g(t)
$$

With

$$
1 \cdot\left[\tau(t)>\tau_{p l}\right]= \begin{cases}1 & \text { if } \tau(t)>\tau_{p l} \\ 0 & \text { if } \tau(t)=\tau_{p l}\end{cases}
$$

The cost function $C(t)$, at time $t$, which is composed by the inventory cost, transportation cost, lost sales cost and late delivery cost, is given by:

$$
C(t)=c s \cdot x(t)+c s^{-} \cdot L(t)+c t \cdot g(t)+c t r \cdot r(t)
$$


where:

cs : unit inventory cost;

$c t:$ unit transportation cost;

$c s^{-}$: unit lost sales cost;

ctr : penalty cost for late delivery per unit product.

The expected average cost, denoted by $J(h)$ depending on $h$ is given by :

$$
J(h)=\lim _{T \rightarrow \infty} \frac{1}{T} \cdot E\left[\int_{0}^{T} C(t) \cdot \mathrm{d} t\right]
$$

$\forall t \in[0, T]$ with $T$ the total simulation time

\section{Application of the IPA Method on the Stochastic Fluid Model}

In this section, we turn our attention, to applying the IPA method to the stochastic fluid model. The IPA is an approach intended to estimate gradients of performances metric with respect to some parameters of interest. This method consists of observing and analyzing two sample paths, one is the nominal sample path $(x(t))$, and the other is the perturbed sample path $\left(x^{\delta}(t)\right)$ (Fig. 2). We assumed that the optimal inventory level is increased by a perturbation, denoted by $\delta$. In this paper, we consider $\delta>0$ and we evaluate the resulting changes in the cost function using geometric arguments (similar results could be easily obtained for $\delta<0$ ). The optimal inventory level of the perturbed sample path $\left(x^{\delta}(t)\right)$ is $h+\delta$.

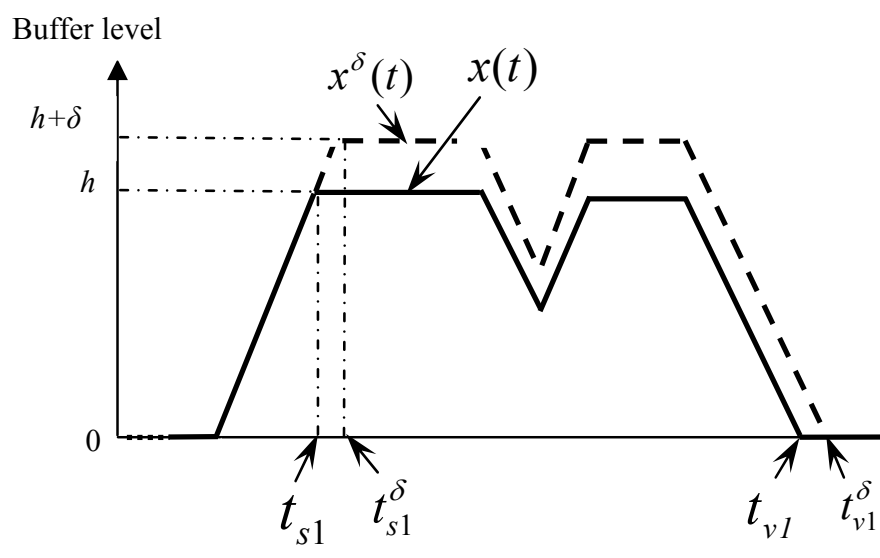

Fig. 2 Buffer level of the perturbed and nominal paths.

\subsection{Study of Buffers Level Trajectory}

We assumed that the optimal buffer level $h$ is increased by a positive perturbation, denoted by $\delta$. Indeed, when the buffer becomes full, a lag denoted by $\phi(t)$ (with $0<\phi(t) \leq \delta$ ) begins to take place between the nominal and perturbed trajectory and increases until it reaches the value of $\delta$.

The following assumptions are considered:

- For comparing the both sample paths, the same distribution of random variables (times to failure, times to repair and customer demands) is used;

- The maximal production is the same for both sample paths, so the number of parts increases in an equivalent way.

The following notations are used:

$x^{\delta}(t)$ : The buffer level for the perturbed path;

$u^{\delta}(t)$ : The production rate at time $t$ for the perturbed path;

$g^{\delta}(t)$ : The number of transported products at time $t$ for the perturbed path;

$D^{0 \delta}(t)$ : The number of unsatisfied demands per unit time for the perturbed trajectory;

$L^{\delta}(t)$ : The number of unsatisfied demands at time $t$ for the perturbed trajectory;

$r^{\delta}(t)$ : The number of products arriving after the planned delivery time at time $t$ for the perturbed trajectory;

$t_{s j}: j^{\text {th }}$ instant for which the buffer on the nominal

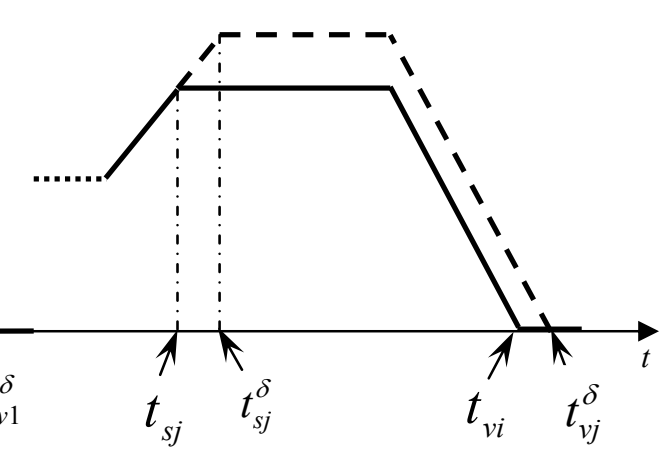


path becomes full and causes a lag between the disturbed path and the nominal path.;

$t_{s j}^{\delta}: j^{\text {th }}$ instant for which the buffer on the disturbed path becomes full;

$t_{v j}^{\delta}: j^{\text {th }}$ instant for which the perturbed trajectory and the nominal trajectory merge;

$t_{v j}$ : is the last instant between $t_{s j}$ and $t_{v j}^{\delta}$ for which the buffer becomes empty on the nominal trajectory.

In the following Fig. 2, we give an example.

Let $[0, T]$ be the finite horizon. In this interval, we consider the trajectories of $x(t)$ and $x^{\delta}(t)$. The interval $[0, T]$ is divided into two alternating periods: the first when $t \in\left[t_{s j}, t_{v j}^{\delta}\right]$ and the other when $t \in\left[t_{v j}^{\delta}, t_{s(j+1)}\right]$. For these intervals, the perturbed and the nominal paths will be analysed and compared. We will use these periods in the following lemmas and theorems.

Lemma 1 shows that at instant $t_{s j}^{\delta}$, the buffer level of the perturbed trajectory becomes full and causes a lag $\phi(t)=\delta$ between the nominal and perturbed trajectory. We will use this lemma for the demonstration of the theorem 1 .

Lemma 1: if $t=t_{s j}^{\delta}$ then $x^{\delta}\left(t_{s j}^{\delta}\right)=x\left(t_{s j}^{\delta}\right)+\delta$.

Theorem 1 shows that the buffer level of the perturbed path is equal to nominal path plus the perturbation when $t \in\left[t_{s j}, t_{v j}^{\delta}\right]$, these periods correspond to the achievement of a buffer full for the perturbed path.

The proofs of following theorems are logically similar to which in Refs. [32, 33].

Theorem 1: If $x^{\delta}=x(0)$ and $t \in\left[t_{s j}, t_{v j}^{\delta}\right]$, then $x^{\delta}(t)=x(t)+\phi(t)$ with $0<\phi(t) \leq \delta$.

The following lemma shows that at instant $t_{v j}^{\delta}$, the buffer level of the perturbed trajectory becomes empty and annuls the lag between the nominal and perturbed trajectory. We will use this lemma in the demonstration of Theorem 2 .

Lemma 2: If $t=t_{v j}^{\delta}$ then $x^{\delta}\left(t_{v j}^{\delta}\right)=0=x\left(t_{v j}^{\delta}\right)$.
Theorem 2 shows that the buffer level of the nominal path and the perturbed path are equal when $t \in\left[t_{v j}^{\delta}, t_{s(j+1)}\right]$, these periods correspond to the buffer building.

Theorem 2: If $x^{\delta}(0)=x(0)$, then $x^{\delta}(t)=x(t)$ for all $t \in\left[t_{v j}^{\delta}, t_{s(j+1)}\right]$.

Theorem 3 shows that the number of materials transported of the perturbed path is equal to nominal path plus the perturbation when $t \in\left[t_{v j}, t_{v j}^{\delta}\right]$.

Theorem 3: If $t \in\left[t_{v j}, t_{v j}^{\delta}\right]$ and $d(t)>0$ then $g^{\delta}(t)=g(t)+\phi(t)$.

Theorem 4 shows that the number of materials transported of the nominal path and of the perturbed path is equal when $t \in[0, T] /\left[t_{v j}, t_{v j}^{\delta}\right]$.

Theorem 4: If $t \in[0, T] /\left[t_{v j}, t_{v j}^{\delta}\right] \quad$ then $g^{\delta}(t)=g(t)$.

Theorem 5 shows that the number of products arriving after the planed delivery time (i.e., $\tau(t)>\tau_{p l}$ ) of the perturbed path is equal to nominal path plus the perturbation when $t \in\left[t_{v j}, t_{v j}^{\delta}\right]$.

Theorem 5: If $t \in\left[t_{v j}, t_{v j}^{\delta}\right]$ and $d(t)>0$ then $r^{\delta}(t)=r(t)+1 \cdot\left[\tau(t)>\tau_{p l}\right] \cdot \phi(t)$.

Theorem 6 shows that the number of products arriving after the planned delivery time of the nominal path and of the perturbed path is equal when $t \in[0, T] /\left[t_{v j}, t_{v j}^{\delta}\right]$.

Theorem 6: If $t \in[0, T] /\left[t_{v j}, t_{v j}^{\delta}\right] \quad$ then $r^{\delta}(t)=r(t)$.

In what follows, we will determine the IPA estimators and will prove their unbiasedness.

\subsection{IPA Estimators}

The expected value of the sample path derivatives obtained by simulation is used instead of the derivative of the expected cost. Therefore, we need to establish the unbiasedness of the gradient estimators. For this, we just have to prove that $E\left(\frac{d\left(J_{a}(h)\right)}{d h}\right)=\frac{d\left(E\left(J_{a}(h)\right)\right)}{d h}$ for every $h$ in $H$, 
where $H$ is the set of possible values of $h$.

The average cost of the perturbed trajectory is given by:

$$
J(h+\delta)=\lim _{T \rightarrow \infty} \frac{1}{T} \cdot E\left[\int_{0}^{T} C^{\delta}(t) \cdot \mathrm{d} t\right]
$$

With

$C^{\delta}(t)=c s \cdot x^{\delta}(t)+c t \cdot g^{\delta}(t)+c s^{-} \cdot L^{\delta}(t)+c t r \cdot r^{\delta}(t)(11)$

The sampled estimation for the expected average cost of the nominal path is given by:

$$
J_{a}(h)=\frac{1}{T} \cdot E\left[\int_{0}^{T} C(t) \cdot d t\right]
$$

The sampled estimation for the expected average cost of the perturbed path is given by:

$$
J_{a}^{\delta}(h)=\frac{1}{T} \cdot E\left[\int_{0}^{T} C^{\delta}(t) \cdot d t\right]
$$

We determine the IPA estimators of the cost function by computing the difference between the perturbed average cost and the nominal average cost.

The difference between the perturbed average cost and the nominal average cost is given by:

$$
\begin{aligned}
& J_{a}^{\delta}(h+\delta)-J_{a}(h)=\frac{1}{T} \cdot E\left[\int_{0}^{T}\left(C^{\delta}(t)-C(t)\right) \cdot d t\right] \\
& J_{a}^{\delta}(h+\delta)-J_{a}(h)= \\
& \frac{1}{T} \cdot E\left[\int_{0}^{T} c s \cdot\left(x^{\delta}(t)-x(t)\right)+c t \cdot\left(g^{\delta}(t)-g(t)\right)+\right. \\
& \left.\left.c s^{-} \cdot\left(L^{\delta}(t)-L(t)\right)+c t r \cdot\left(r^{\delta}(t)-r(t)\right)\right) \cdot d t\right]
\end{aligned}
$$

We assume that in the interval $[0, T]$, we have $m$ intervals $\left[t_{s j}, t_{v j}^{\delta}\right]$.

For $\quad \int_{0}^{T} c s \cdot\left(x^{\delta}(t)-x(t)\right) \cdot d t, \quad$ we have $x^{\delta}(t)=x(t)+\delta \quad$ when $\quad t \in\left[t_{s j}, t_{v j}^{\delta}\right] \quad$ and $x^{\delta}(t)=x(t)$, when $t \in\left[t_{v j}^{\delta}, t_{s(j+1)}\right]$ (Theorems 1 and 2) then we have:

$$
\begin{aligned}
& \int_{0}^{T} c s \cdot\left(x^{\delta}(t)-x(t)\right) \cdot d t= \\
& c S \cdot\left(\sum_{j=1}^{j=m_{t_{s j}}^{t^{\delta}}} \int_{t_{j j}}\left(x^{\delta}(t)-x(t)\right) \cdot d t\right)=c s \cdot\left(\sum_{j=1}^{j=m_{t_{s j}}^{t^{\delta}}} \int_{j, j}(\delta) \cdot d t\right)
\end{aligned}
$$

For $\quad \int_{0}^{T} c t \cdot\left(g^{\delta}(t)-g(t)\right) \cdot d t \quad$ we have $g^{\delta}(t)=g(t)+\delta$ when $t \in\left[t_{v j}, t_{v j}^{\delta}\right]$ (Theorem 3) and we have $g^{\delta}(t)=g(t)$ when $t \in[0, T] /\left[t_{v j}, t_{v j}^{\delta}\right]$ (Theorem 4), then we have:

$$
\begin{gathered}
\int_{0}^{T} c t \cdot\left(g^{\delta}(t)-g(t)\right) \cdot d t= \\
\left.c t \cdot\left(\sum_{j=1}^{j=m_{t_{v j}}^{t_{v j}^{\delta}}} \int_{t^{\prime}}^{\delta}(t)-g(t)\right) \cdot d t\right)=c t \cdot\left(\sum_{j=1}^{j=m} \int_{t_{v j}}^{t_{v j}^{\delta}}(\delta) \cdot d t\right)
\end{gathered}
$$

For $\quad \int_{0}^{T} c s^{-} \cdot\left(L^{\delta}(t)-L(t)\right) \cdot d t, \quad$ when $\quad t \quad \in$

$[0, T] /\left[t_{v j}, t_{v j}^{\delta}\right]$, the demands are satisfied for the two types of trajectories, then we have $L^{\delta}(t)=L(t)$. When $t \in\left[t_{v j}, t_{v j}^{\delta}\right]$, we have, the number of satisfied products is equal to the number of products in the buffer and then we have $x^{\delta}(t)-x(t)=\delta$. Contrariwise, the difference between the number of unsatisfied products for the perturbed trajectory and that for the nominal trajectory is equal to the opposed for the case of satisfied products, thus $L^{\delta}(t)-L(t)=-\delta$ :

$$
\begin{aligned}
& \int_{0}^{T} c s^{-} \cdot\left(L^{\delta}(t)-L(t)\right) \cdot d t= \\
& c s^{-} \cdot\left(\sum_{j=1}^{j=m_{t j} t_{v_{j}}^{\delta}}\left(L^{\delta}(t)-L(t)\right) \cdot d t\right)=c s^{-} \cdot\left(\sum_{j=1}^{j=m_{t j} t_{v j}^{\delta}}(-\delta) \cdot d t\right)
\end{aligned}
$$

For $\quad \int_{0}^{T} c t r \cdot\left(r^{\delta}(t)-r(t)\right) \cdot d t, \quad$ we have $r^{\delta}(t)=r(t)+1 \cdot\left[\tau(t)>\tau_{p l}\right] \cdot \phi(t) \quad($ Theorem 5$)$ then

$$
\int_{0}^{T} c t r \cdot\left(r^{\delta}(t)-r(t)\right) \cdot d t=
$$


$\int_{0}^{T} c t r \cdot\left(1 \cdot\left[\tau(t)>\tau_{p l}\right] \cdot \phi(t)\right) \cdot d t$ and according to

Theorems 5 and 6 :

$$
\begin{aligned}
& \int_{0}^{T} c t r \cdot\left(1 \cdot\left[\tau(t)>\tau_{p l}\right] \cdot \phi(t)\right) \cdot d t= \\
& c t r \cdot 1 \cdot\left[\tau(t)>\tau_{p l}\right] \cdot\left(\sum_{j=1}^{j=m_{t y j}^{t_{v j}^{\delta}}} \int_{t_{v j}}(\delta) \cdot d t\right) \\
& J_{a}^{\delta}(h+\delta)-J_{a}(h)= \\
& \frac{1}{T} \cdot E\left[c s \cdot\left(\sum_{j=1}^{j=m_{t_{s j}}^{t_{v j}^{\delta}}}(\delta) \cdot d t\right)+c t \cdot\left(\sum_{j=1}^{j=m^{\prime}} \int_{t_{v j}}^{t_{v j}^{\delta}}(\delta) \cdot d t\right)+\right. \\
& c s^{-} \cdot\left(\sum_{j=1}^{j=m_{t_{v j}}^{t_{v j}^{\delta}}}(-\delta) \cdot d t\right)+c t r \cdot\left(\sum_{j=1}^{j=m_{t_{v j}}^{t_{v j}^{\delta}}}(\delta) \cdot 1 \cdot\left[\tau(t)>\tau_{p l}\right] \cdot d t\right)
\end{aligned}
$$

We note $T_{1}=\sum_{j=1}^{j=m}\left(t_{v j}^{\delta}-t_{s j}\right), \quad T_{2}=\sum_{j=1}^{j=m}\left(t_{v j}^{\delta}-t_{v j}\right)$ and $T_{3}=\sum_{j=1}^{j=m}\left(1 \cdot\left[\tau\left(t_{v j}\right)>\tau_{p l}\right]\right) \cdot\left(t_{v j}^{\delta}-t_{v j}\right)$ with $T_{1}+$ $T_{2}+T_{3} \leqslant T$.

$$
\begin{aligned}
& J_{a}^{\delta}(h+\delta)-J_{a}(h)= \\
& \frac{1}{T} \cdot E\left[T_{1} \cdot c s+T_{2}\left(c t-c s^{-}\right)+T_{3} \cdot c t r\right] \cdot \delta
\end{aligned}
$$

The gradients of the cost function are defined as follow:

$$
\begin{aligned}
& \frac{\partial J_{a}(h)}{\partial h}=E\left[c s \cdot \frac{T_{1}}{T}+\frac{T_{2}}{T} \cdot\left(c t-c s^{-}\right)+\frac{T_{3}}{T} \cdot c t r\right] \\
& \frac{\partial J_{a}(h)}{\partial h}= \\
& E\left[G^{\prime}(h) \cdot c s+W^{\prime}(h) \cdot\left(c t-c s^{-}\right)+S^{\prime}(h) \cdot c t r\right]
\end{aligned}
$$

with $G^{\prime}(h)=\frac{T_{1}}{T}, W^{\prime}(h)=\frac{T_{2}}{T}$ and $S^{\prime}(h)=\frac{T_{3}}{T}$.

For making these estimators useful in practice, the unbiasedness should be proved.

Theorem 7: The gradient estimator of the average cost is unbiased.

Before presenting our numerical results, we present an algorithm (IPA estimation algorithm) for determining the IPA estimators which will be used thereafter in an optimisation algorithm in Table 1.

Let $Z, Q$ and $F$ be the IPA estimators (parameters) which will be used in the following algorithm, with $G^{\prime}(h)=\frac{Z}{T}, W^{\prime}(h)=\frac{Q}{T}$ and $S^{\prime}(h)=\frac{F}{T}$.

For determining the optimal buffer level, we use an optimisation algorithm, which is bisection algorithm. The choice of this type of algorithm is explained by the fact that this algorithm is widely used for optimising a convex function (cost function) with the use of a gradient method such as the IPA method. Furthermore, in the literature this type of algorithm is often used in the case of a single decision variable, which is our case. Indeed, our optimisation algorithm computes at every step the gradients estimation of the cost function by using the IPA estimation algorithm, which determines the estimators $R$ and $S$. According to the sign of the gradients estimation, the proposed algorithm determines the new values of the interval bounds (new sub-interval) and repeats all steps until the difference between the upper and lower bound becomes very small, thus the value of the optimal buffer level is found. In what follows, we present our optimisation algorithm in Table 2.

Table 1 IPA estimation algorithm.

Beginning $Z=0, Q=0, F=0, t=0, q=0, l=0, w=0$

Do

If $t=t_{s j}^{\delta}$ then $q=t_{s j}^{\delta}$.

Advance $t$.

If $t=t_{v j}$ then $l=t_{v j}$.

Advance $t$.

If $t=t_{v j}^{\delta}$ then $w=t_{v j}^{\delta}$.

$Z=Z+(w-q)$.

$Q=Q+(w-l)$.

If $\tau\left(t_{v j}\right)>\tau_{p l}$ then $F=F+(w-l)$.

$q=l=w=0$.

Advance $t$.

While $t<T$

End 
Table 2 Optimisation algorithm.

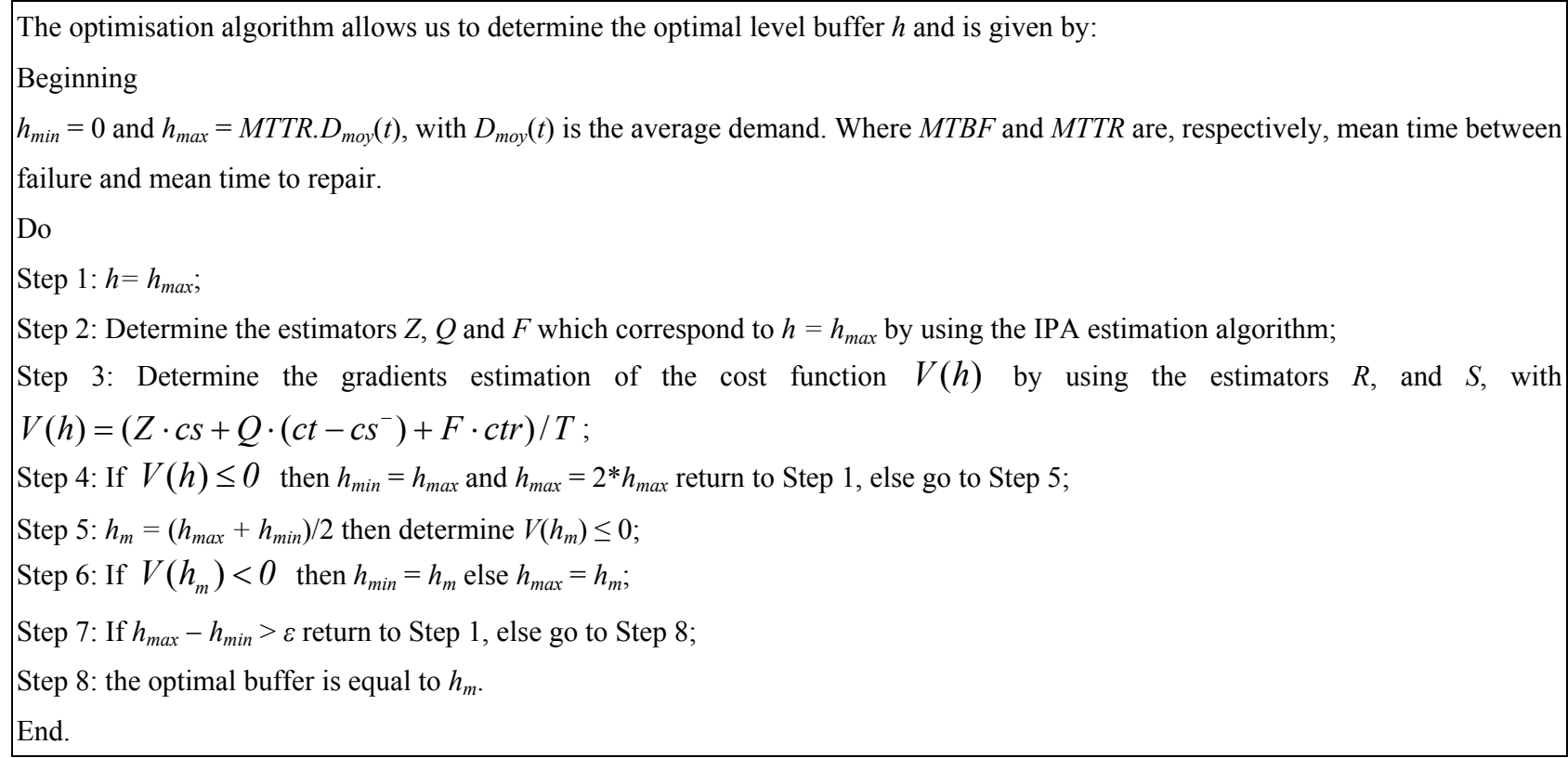

\section{Numerical Results}

In this section, we use the IPA estimators in an optimization algorithm, which allows us to determine the value of $h$. Such as the cost function depends on the value of the unit costs $c s, c t, c s^{-}$and $c t r$, the value of $h$ which minimizes this cost function will certainly also depend on these unit costs. We are interested in this study to the value of $c s^{-}$and ctr. Therefore, we fix the values of $c s$, $c t$ and we vary the values of $c s^{-}$and $\mathrm{ctr}$.

The following parameters are used for the simulation:

$$
U=2 \text { products/time unit }
$$

The average of demands $d(t)$ over the interval $[0, T]$ is 0.8 products/time unit. For the demand event the boundaries of the uniform distribution are 1 (minimum) and 4 (maximum);

The total simulation time is equal to $T=10 \mathrm{E}+07$ time units;

The times to failure or repair are given by exponential distribution, the mean time between failures $M T B F$ is equal to 2.3 and the mean time to repair MTTR is equal to 1.1 ;

The planned delivery time $\tau_{p l}$ is equal to 2 , the delivery time $\tau(t)$ is generated according to a truncated normal distribution, the normal distribution gives same value out the interval $[1,3]$, the truncated normal distribution gives bounded value between 1 and 3 .

Simulation results are presented in the following table to show the impact of the unit costs $\mathrm{Cs}^{-}$and $\mathrm{ctr}$ on the value of $h$. Furthermore, to evaluate the simulation results we simulated the stochastic fluid model for an example of the unit costs values ( $c s, c t$, $\mathrm{Cs}^{-}$and $\left.\mathrm{ctr}\right)$.

In the following, in Tables 3 , we present simulation examples of the stochastic fluid model according to the unit costs values:

$$
\text { Example }(c s=5 \text { and } c t=10)
$$

In this example, we see that the more the unit cost ctr becomes important comparing to $\mathrm{cs}^{-}$; the more the value of $h$ decreases. Turki et al. [31] showed that in the case of constant delivery time, $\mathrm{Cs}^{-}$has the most important impact on the value of $h$ comparing to $c t$ and $c s$, and when $c s^{-}$increases the optimal buffer level $h$ increases too. In this paper, we considered a stochastic delivery time, which generates late delivery cost. Therefore, when when ctr becomes important comparing to $\mathrm{CS}^{-}$normally the late delivery cost becomes important comparing to lost sale cost. Thus, 
Table 3 Impact of unit costs $\left(c s^{-}\right.$and $\left.c t r\right)$ on $h$ (Case 1).

\begin{tabular}{lll}
\hline$c s^{-}$ & $c t r$ & $h$ \\
\hline 50 & 1 & 4.358 \\
50 & 10 & 3.885 \\
50 & 20 & 3.183 \\
50 & 30 & 2.852 \\
50 & 40 & 1.788 \\
50 & 50 & 1.253 \\
40 & 1 & 3.741 \\
40 & 10 & 2.924 \\
40 & 20 & 2.223 \\
40 & 30 & 2.752 \\
40 & 40 & 1.491 \\
40 & 50 & 0.818 \\
30 & 1 & 3.081 \\
30 & 10 & 2.481 \\
30 & 20 & 1.875 \\
30 & 30 & 1.104 \\
30 & 40 & 0.785 \\
30 & 50 & 0.391 \\
20 & 1 & 2.252 \\
20 & 10 & 1.634 \\
20 & 20 & 1.092 \\
20 & 30 & 0.645 \\
20 & 40 & 0.204 \\
20 & 50 & 0.092 \\
\hline
\end{tabular}

the impact of $c s^{-}$on the value of $h$ becomes less important in the presence of stochastic delivery time. Then the value of $h$, which minimizes the total cost, of course decreases as the unit cost ctr increases. However, these numerical results, which are obtained by a simulation based on IPA are computed in small simulation time. For example, a simulation based on bisection technique takes six hours for determining the value of the optimal buffer level. Besides, the simulation based on IPA takes three minutes for determining the value of the optimal buffer level. Then, the advantage of IPA method is that the simulation based on IPA allows reducing the simulation time comparing to classical simulation methods.

\section{Conclusions}

In this paper, a manufacturing supply chain system composed by a single-product machine, a buffer and a stochastic demand is considered. The demand is lost when it is unsatisfied. The delivery time is random. The times to failure and times to repair are random variables with exponential distribution. A hedging point policy taken into account the stochastic delivery time, machine failures and random demands is defined. A stochastic fluid model with explicit modeling of the planned delivery time between the producer and the customer is proposed. The IPA method is applied; the stochastic fluid model and the buffer level trajectories are studied and analyzed. Two alternating and repetitive periods appear. The first period is when the perturbed and nominal paths are equal: the buffer is build. The second period is when the perturbed path is higher than the nominal path: the buffer full is reached. The infinitesimal perturbation analysis estimators are determined and shown to be unbiased. These estimators are then implemented in an optimization algorithm for determining the optimal buffer level. The advantage of IPA method is that the simulation based on IPA allows reducing the simulation time comparing to classical simulation methods. The impact of the unit costs $c s^{-}$and $c t r$ on the value of $h$ is studied. Indeed, when the value of $c t r$ increases, $h$ decreases.

For future research, we will study the impact of the planned delivery time on $h$. Also, we will consider a more complex system with multi-stage and multi-product machines. We will consider also more than one customer with random demands for each one.

\section{References}

[1] Markou, M., and Panayiotou, C. G. 2007. "Optimisation of Discrete Event System Parameters Using SFM-Based Infinitesimal Perturbation Analysis Estimates.” Presented at the 46th IEEE Conference on Decision and Control, New Orlean, USA.

[2] Turki, S., and Rezg, N. 2014. "Perturbation Analysis for Discrete Flow Model: Optimization of a Manufacturing-Remanufacturing System." Presented at the 2014 IEEE International Conference on Systems, Man, and Cybernetics (SMC2014), San Diego, CA ,USA.

[3] Turki, S., Hennequin, S., and Sauer, N. 2013. "Perturbation Analysis for Continuous and Discrete Flow Models: A Study of the Delivery Time Impact on the Optimal Buffer Level." International Journal of 
Production Research 51 (13): 4011-44.

[4] Turki, S., Bistorin, O., and Rezg, N. 2013. "Infinitesimal Perturbation Analysis Based Optimization for a Manufacturing-Remanufacturing System." Presented at the 18th IEEE Conference on Emerging Technologies and Factory Automation (ETFA 2013), Cagliari, Italy.

[5] Manitz, M. 2008. "Queuing-Model Based Analysis of Assembly Lines with Finite Buffers and General Service Times." Computers \& Operations Research 35 (8): 2520-36.

[6] Egerstedt, M., and Wardi, Y. 2002. "Multi-process Control Using Queuing Theory." Presented at the IEEE Conference on Decision and Control, Vol. 2, 1991-99, Las Vegas, USA.

[7] Kumaran, K., and Mitra, D. 1998. "Performance and Fluid Simulations of a Novel Shared Buffer Management System." In Proceedings of IEEE INFOCOM 11 (1): 43-75.

[8] Turki, S., Bistorin, O., and Rezg, N. 2014. "Infinitesimal Perturbation Analysis for Optimal Production Control in a Reverse Logistic System with Different Demands." Presented at the 19h IEEE Conference on Emerging Technologies and Factory Automation (ETFA 2014), Barcelona, Spain.

[9] Tzenova, E. I., Adan, I. J. B. F., and Kulkarni, V. G. 2008. "Output Analysis of Multiclass Fluid Models with Static Priorities." Performance Evaluation 65 (1): 71-81.

[10] Turki, S., Hennequin, S., and Sauer, N. 2012. "Perturbation Analysis Based-Optimization for Discrete Flow Model: A Failure-Prone Manufacturing System with Constant Delivery Time and Stochastic Demand." Int. J. Advanced Operations Management 4 (1/2): 124-53.

[11] Turki, S., Bistorin, O., and Rezg, N. 2013. "Optimization of a Manufacturing System with Delivery Time and Withdrawal Right." Presented at International Conference on Production and Manufacturing Engineering (ICPME 2013), Tokyo, Japan.

[12] Turki, S., Bistorin, O., and Rezg, N. 2014. "Optimization of Stochastic Fluid Model Using Perturbation Analysis: A Manufacturing-Remanufacturing System with Stochastic Demand and Stochastic Returned Products." Presented at the 11th IEEE International Conference on Networking, Sensing and Control (ICNSC14), Miami, FL, USA.

[13] Turki, S., and Rezg, N. 2016. "Study of a Manufacturing System with Transport Activities in Urban Area." Presented at the 14th IFAC Symposium on Control in Transportation Systems CTS 2016, Vol. 49, 419-23. Istanbul, Turkey.

[14] Turki, S., and Rezg, N. 2016. "Optimal Production Control of a Manufacturing-Remanufacturing System with Delivery Activity." Presented at the 8th IFAC
Conference on Manufacturing Modelling, Management and Control MIM 2016, Vol. 49, 1233-8. Troyes, France.

[15] Guiras., Z., Turki, S., Rezg, N., and Dolgui, A. 2016. "Optimal Supply Planning for Two-Levels Assembly System with Stochastic Lead-Times and Maintenance Actions." Presented at the Industrial Engineering and Engineering Management (IEEM), 2016 IEEE International Conference.

[16] Hajej, Z., Turki, S., and Rezg, N. 2015. "Modelling and Analysis for Sequentially Optimising Production, Maintenance and Delivery Activities Taking into Account Product Returns." International Journal of Production Research 53 (15): 4694-719.

[17] Yao, C., and Cassandras, C. G. 2011. "Perturbation Analysis and Optimization of Multiclass Multiobjective Stochastic Flow Models." Discrete Event Dynamic Systems 21: 219-56.

[18] Ho, Y. C., Eyler, M. A., and Chien, T. T. 1979. "A Gradient Technique for General Buffer Storage Design in a Production Line.” I. J. of Production Research 17 (6): 557-80.

[19] Ho, Y. C. 1985. "A Survey of the Perturbation Analysis of Discrete Event Dynamic Systems." Annals of Op. Research 3: 393-402.

[20] Ho, Y. C., and Cao, X. R. 1991. Perturbation Analysis of Discrete Event Dynamic Systems. Norwell, MA: Kluwer.

[21] Xia, L., and Cao, X. R. 2012. "Performance Optimization of Queueing Systems with Perturbation Realization." European Journal of Operational Research 218: 293-304.

[22] Panayiotou, C. G., and Cassandras, C. G. 2008. "IPA for Delay Threshold Violation Using Stochastic Fluid Models." In Proceedings of the 9th International Workshop on Discrete Event Systems, 162-7. Göteborg, Sweden.

[23] Markou, M., and Panayiotou, C. G. 2011. "On-line Optimization and Control of the Buffer Sizes in a Cellular Network Communication System." Presented at the 18th International Conference on Telecommunications, Ayia Napa, Cyprus.

[24] Panayiotou, C. G., and Cassandras, C. G. 2006. "Infinitesimal Perturbation Analysis and Optimisation for Make-to-Stock Manufacturing Systems Based on Stochastic Fluid Models." Discrete Event Dynamic System 16 (1): 109-42.

[25] Yao, C., and Cassandras, C. G. 2012. "A Solution to the Optimal Lot-Sizing Problem as a Stochastic Resource Contention Game.” IEEE Transactions on Automation Science and Engineering 9: 250-64.

[26] Ayed, S., Hajej, Z., Turki, S., and Rezg, N. 2016. "FPA Method for Optimal Production Planning under 
Availability/Degradation Machine and Subcontracting Constraint." International Journal of Production Research, 1-14. DOI:10.1080/00207543.2016.1165879.

[27] Heidergott, B. 2000. "Customer-Oriented Finite Perturbation Analysis for Queueing Networks.” D.E.D.S.: Theory and Applications 10: 201-32.

[28] Mokou, P. Y., and Porter, B. 2005. "Evolutionary Optimisation of Hedging Points for Unreliable Manufacturing Systems." International Journal $A d v$ Manufacturing Technologies 28 (1-2): 205-14.

[29] Wang, Z., and Yugang, Y. 2012. "Robust Production Control Policy for a Single Machine and Single Part-Type Manufacturing System with Inaccurate Observation Of Production Surplus." IIE Transactions 44 (12): 1061-82.

[30] Akella, R., and Kummar, P. R. 1986. "Optimal Control of Production Rate in Failure Prone Manufacturing System.”
IEEE Transaction on Automatic Control 31 (2): 116-26.

[31] Turki, S., Hennequin, S., and Sauer, N. 2009. "Performances Evaluation of a Failure-Prone Manufacturing System with Time to Delivery and Stochastic Demand." Presented at the INCOM 09, 13th IFAC Symposium On Information Control Problems in Manufacturing. Vol13. Moscow, Russia.

[32] Turki, S., and Rezg, N. 2016. "Unreliable Manufacturing Supply Chain Optimisation Based on an Infinitesimal Perturbation Analysis." International Journal of Systems Science: Operations \& Logistics, 1-20. DOI: 10.1080/23302674.2016.1212427.

[33] Turki, S., and Rezg, N. 2016. "Study of a Manufacturing System with Transport Activities in Urban Area." In Proceedings of the 14th IFAC Symposium on Control in Transportation Systems CTS 2016, Vol. 49, 419-23. Istanbul, Turkey. 